\title{
An attempt to calibrate core overshooting using the seismic properties of low-mass stars
}

S. Deheuvels ${ }^{1,2, a}$, V. Silva Aguirre ${ }^{3}$, M. S. Cunha ${ }^{4,5}$, T. Appourchaux ${ }^{6}$, J. Ballot ${ }^{1,2}$, I. Brandão ${ }^{4,5}$, Y. Lebreton $^{7,8}$, and E. Michel ${ }^{9}$

1 Université de Toulouse; UPS-OMP; IRAP; Toulouse, France

2 CNRS; IRAP; 14, avenue Edouard Belin, F-31400 Toulouse, France

3 Stellar Astrophysics Centre, Department of Physics and Astronomy, Aarhus University, Ny Munkegade 120, DK-8000 Aarhus C, Denmark

4 Instituto de Astrofísica e Ciências do Espaço, Universidade do Porto, CAUP, Rua das Estrelas, 4150762 Porto, Portugal

5 Centro de Astrofísica e Faculdade de Ciências, Universidade do Porto, Rua das Estrelas, 4150-762 Porto, Portugal

6 Univ. Paris-Sud, Institut d'Astrophysique Spatiale, UMR 8617, CNRS, Bâtiment 121, 91405 Orsay Cedex, France

7 Observatoire de Paris, GEPI, CNRS UMR 8111, F-92195 Meudon, France

8 Institut de Physique de Rennes, Université de Rennes 1, CNRS UMR 6251, F-35042 Rennes, France

9 LESIA, CNRS UMR 8109, Université Pierre et Marie Curie, Université Denis Diderot, Observatoire de Paris, 92195 Meudon, France

\begin{abstract}
The sizes of stellar convective cores remain uncertain because of our poor understanding of the interface between convective and radiative zones. The very high precision of the seismic data provided by the CoRoT and Kepler space missions offers a great opportunity to search for the signature of convective cores in main-sequence stars. We here validate the seismic diagnostic based on the $r_{010}$ ratios, which has been proposed to probe the size of convective cores, and we use it on a sample of 24 specially chosen Kepler targets. We thus constrain the extension of the core in 14 targets and find a tendency of the core extension to increase with stellar mass in this mass range. These results will be presented in more detail in a paper in preparation.
\end{abstract}

\section{Introduction}

Several physical mechanisms could extend the size of convective cores beyond the Schwarzschild boundary, such as the overshooting of convective elements in the stable interior, semi-convection, rotational mixing... These processes are still not satisfactorily understood by theory. Their combined effects are often modeled in 1D stellar models as a simple extension of the mixed convective core over a distance $d_{\mathrm{ov}}=\alpha_{\mathrm{ov}} \times \min \left(H_{p}, R_{\mathrm{c}}\right)$, where $H_{p}$ is the pressure scale height at the edge of the core and $R_{\mathrm{c}}$ is the radius of the convective core. As is usually done, we will refer to $d_{\mathrm{ov}}$ as the overshooting distance, even though overshooting may not be the only mechanism at work. This model is of course simplistic but its simplicity only reflects our current ignorance about the interface between convective and radiative regions.

The detection of solar-like oscillations in several hundreds of main-sequence stars by the space missions CoRoT and Kepler makes it opportune to try using seismology to estimate the extension of convective cores. The boundary of the convective core generates a glitch in the sound speed velocity,

a e-mail: sebastien.deheuvels@irap.omp.eu

This is an Open Access article distributed under the terms of the Creative Commons Attribution License 4.0, which permits unrestricted use, distribution, and reproduction in any medium, provided the original work is properly cited. 


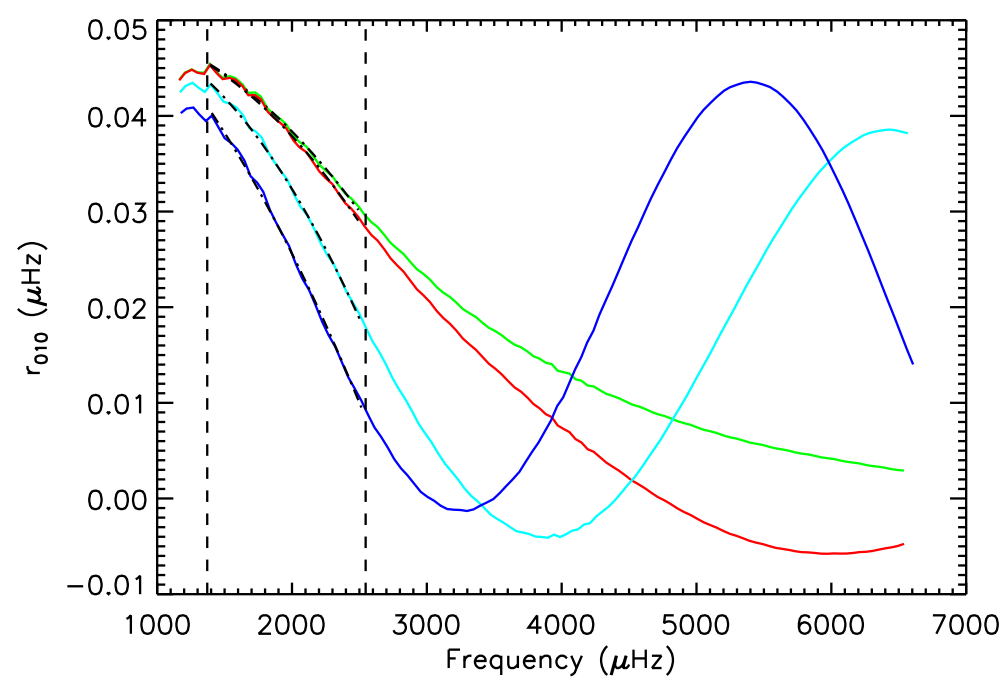

Fig. 1. Variations in the ratio $r_{010}$ as a function of frequency for models of $1.2 M_{\odot}$ at solar metallicity and an age of $3.5 \mathrm{Gyr}$ for different amounts of overshooting (green: $\alpha_{\mathrm{ov}}=0$, red: $\alpha_{\mathrm{ov}}=0.1$, cyan: $\alpha_{\mathrm{ov}}=0.15$, blue: $\alpha_{\mathrm{ov}}=0.2$ ). The vertical dashed lines indicate the frequency interval where solar-like oscillations are expected to be excited. The dot-dashed lines correspond to fits of $2^{\text {nd }}$ order polynomials in this range.

to which acoustic waves are sensitive: it causes the mode frequencies to oscillate as a function of the radial order, with a period that is directly related to the depth of the glitch [4]. Thus, seismology can be used as a tool to measure the size of mixed cores. It has been shown that the small separations built with $l=0$ and $l=1$ modes are particularly sensitive to the core [3,7], and that their ratio to the large separations $\Delta v$ (hereafter referred to as $r_{010}$ ) are almost immune to the so-called near-surface effects [6]. Using this seismic diagnostic, a convective core could already be detected in a Kepler target with 9 months of data [8]. Now, with more than 3 years of Kepler data, more mode frequencies can be estimated with a better precision, and we can hope to bring constraints on the extension of the convective cores.

\section{Testing the seismic diagnostic of $r_{010}$ ratios}

We built a grid of models with three main objectives: (1) to assess the efficiency of the seismic diagnostic given by $r_{010}$ ratios, (2) to determine criteria to select the most promising Kepler targets, and (3) to interpret these data by confronting them to the grid of models that was built and obtain constraints on the amount of core overshooting. The grid was computed using the stellar evolution code CESAM $2 \mathrm{~K}$ and the oscillation code Losc, with masses ranging from 0.9 to $1.5 M_{\odot}$, metallicities from -0.4 to 0.4 dex, and initial helium abundance from 0.26 to 0.30 . Core overshooting was included as a simple extension of the homogeneous convective core over a distance $\alpha_{\mathrm{ov}} H_{p}$, where $H_{p}$ is the pressure scale height and $\alpha_{\mathrm{ov}}$ the overshoot parameter. Models were computed for values of $\alpha_{\mathrm{ov}}$ ranging from 0 to 0.2 .

It should be noted that the period of the oscillation caused by the glitch at the boundary of the core is longer than the frequency range of the observed modes, so only a fraction of the oscillation can be observed. This makes it very hard to derive model-independent information about the core properties, although in certain cases the existence of a convective core can be tested this way $[2,1]$. Here we chose a model-dependent approach. The $r_{010}$ ratios can generally be well approximated by $2^{\text {nd }}$-order polynomials (see Fig. 1). We thus fitted to the $r_{010}$ ratios of each model with polynomials of the type

$$
P(v)=a_{0}+a_{1}(v-\beta)+a_{2}\left(v-\gamma_{1}\right)\left(v-\gamma_{2}\right)
$$

where the parameters $\beta, \gamma_{1}$, and $\gamma_{2}$ were determined to ensure the independence of the $a_{k}$ coefficients. We then used the parameters $a_{k}$ to study the core properties. 

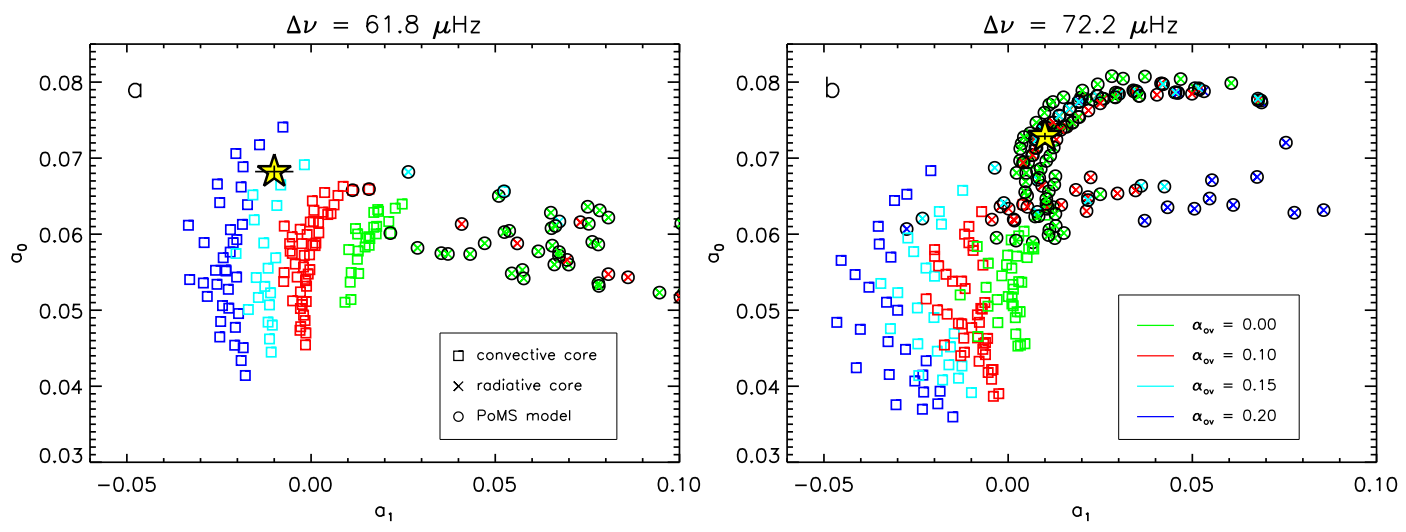

Fig. 2. Location of two stars of the sample in the $\left(a_{1}, a_{0}\right)$ plane (star symbols and black error bars). Models that match the observed large separation, spectroscopic metallicity, and the seismic mass are overplotted.

For observed stars, we have access to a precise estimate of the large separation $\Delta v$, so it is instructive to show models of the grid at a fixed $\Delta v$ in the $\left(a_{1}, a_{0}\right)$ plane. Examples are shown in Fig. 2. This figure clearly shows that constraints can be obtained on

1. the evolutionary status: main sequence (MS) and post main sequence (PoMS) models are well separated in the $\left(a_{1}, a_{0}\right)$ plane

2. the existence or absence of a convective core

3. the size of the convective core if it exists

We insist that a wide range of metallicities has been considered in our grid, so the constraints listed above can be obtained even when the effects of metallicity on the size of the core are taken into account. Such constraints can be obtained only if the star is evolved enough so that the edge of the convective core has had time to build up a $\mu$-gradient, but not too evolved so that the $l=1$ modes do not yet have a mixed behavior (this corresponds approximately to $60 \mu \mathrm{Hz} \lesssim \Delta v \lesssim 110 \mu \mathrm{Hz}$ ).

\section{Measuring the extension of convective cores in Kepler targets}

We used the criteria derived from our grid of models to select among solar-like pulsators observed with Kepler. We thus obtained a set of 24 targets, with masses ranging from 0.9 to $1.4 M_{\odot}$. We used a maximum likelihood estimation (MLE) method to extract the frequencies of the $l=0$ and $l=1$ modes from the power spectra of these stars. We then fitted $2^{\text {nd }}$-order polynomial to the observed $r_{010}$ ratios. We took into account the high level of correlation between the observables.

For each Kepler target, we selected the models of our grid that reproduce the observed large separation $\Delta v$, surface metallicity, and seismic mass, and we compared their location in the $\left(a_{1}, a_{0}\right)$ plane to the one obtained from the observations (yellow stars in Fig. 2). We recall that the $r_{010}$ ratios are nearly insensitive to near-surface effects so a direct comparison between models and observations is possible.

The evolutionary status of the stars can be unambiguously established in most cases: 12 stars of the sample are found to be in the MS (e.g. Fig. 2a), 10 stars in the PoMS (e.g. Fig. 2b), and for only 2 stars of the sample, the evolutionary status remains ambiguous. Among the MS stars, a convective core was clearly detected in 8 targets (e.g. Fig. 2a). As predicted by the grid of models, the position in the $\left(a_{0}, a_{1}\right)$ plane of the stars that have a convective core provides an estimate of the amount of core overshooting. Interestingly, the 8 stars draw a very consistent picture of the extension of convective cores in low-mass stars: all the targets require an extension of the mixed core over at least a small fraction of $H_{p}$, and with our prescription for overshooting the seismic properties of all the targets can be reproduced with $\alpha_{\mathrm{ov}}<0.2$. We insist that seismology provides information about the size of the core at current age. The amounts of overshooting that are quoted here are those required so that the 


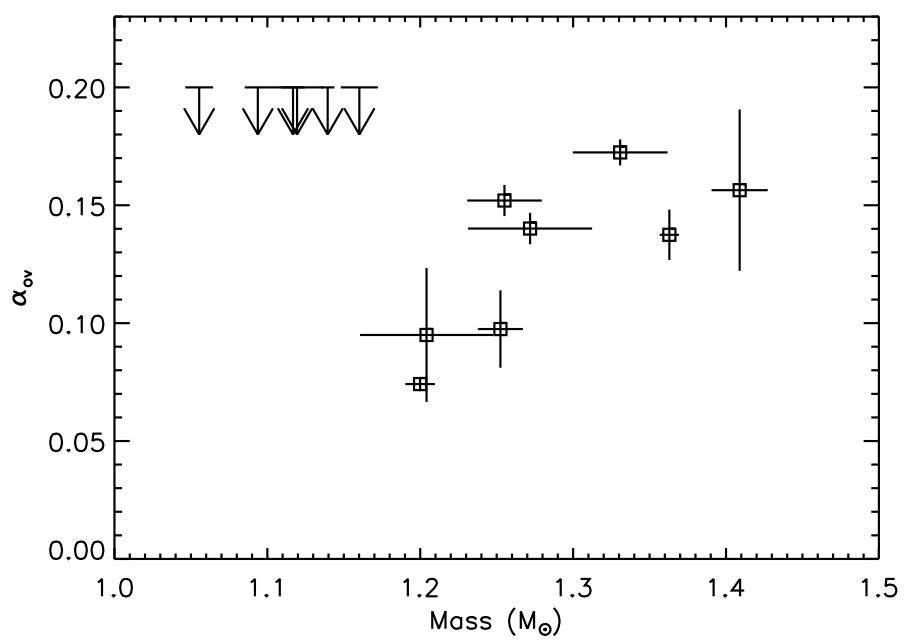

Fig. 3. Amount of core overshooting found for the stars of the sample that have a convective core as a function of the fitted stellar mass. The vertical arrows indicate upper limits of $\alpha_{\mathrm{ov}}$.

evolution code CESAM $2 \mathrm{~K}$ produces cores with appropriate sizes. The reader should be careful that it depends on the prescription for core overshooting, and also likely on the evolution code. In the paper that will present these results, we intend to study this latter dependence.

By performing optimizations using the Levenberg-Marquardt algorithm, we obtained more precise estimates of the amount of overshooting for the stars that have a convective core, as well as better estimates of the stellar mass. During the optimization process, $\alpha_{\mathrm{ov}}$ is left free and can take on values above 0.2. Fig. 3 represents the obtained values of $\alpha_{\mathrm{ov}}$ as a function of the best-fit mass, which interestingly suggest that core overshooting increases with stellar mass. Of course more data points will be required to confirm this tendency.

Information about core overshooting can also be drawn from stars that have no convective core but lie just below the mass limit for having one. Indeed, above a certain amount of core overshooting, the models all develop a convective core and the profile of the $r_{010}$ becomes at odds with the observations. We thus obtained an upper limit of $\alpha_{\mathrm{ov}}<0.2$ for 6 stars of the sample, which is consistent with the constraints obtained for the 8 stars that have a convective core (see Fig. 3).

\section{Conclusion}

By analyzing the seismic data of 24 low-mass Kepler targets, we detected convective cores in 8 of them and were able to estimate the extension of these cores beyond the Schwarzschild boundary. With our prescription for core overshooting and the evolution code $\operatorname{CESAM} 2 \mathrm{~K}$, we found that the seismic properties of these stars were satisfactorily reproduced for amounts of core overshooting ranging from $0.05 \leqslant \alpha_{\mathrm{ov}} \leqslant 0.2$. We also found hints that the amount of core overshooting increases with stellar mass. If this tendency can be confirmed, it could be used to propose a seismic calibration of the extension of convective cores for low-mass stars. To provide a useful calibration, it will be necessary to investigate its dependence on the chosen stellar evolution code (here models were computed exclusively with CESAM $2 \mathrm{~K}$ ). In the end, such a calibration could be used to improve the determination of stellar ages, which is one of the announced goals of the forthcoming Plato mission [5].

\section{References}

1. Brandão, I. M., Cunha, M. S., \& Christensen-Dalsgaard, J., Monthly Notices of the Royal Astronomical Society 438, 1751 (2014) 
2. Cunha, M. S., \& Brandão, I. M., A\&A 529, A10 (2011)

3. Deheuvels, S., Michel, E., Goupil, M. J., et al., A\&A 514, A31 (2010)

4. Gough, D. O., Progress of Seismology of the Sun and Stars 367, 283 (1990)

5. Rauer, H., Catala, C., Aerts, C., et al., Experimental Astronomy 41 (2014)

6. Roxburgh, I. W., \& Vorontsov, S. V., A\&A 411, 215 (2003)

7. Silva Aguirre, V., Ballot, J., Serenelli, A. M., \& Weiss, A., A\&A 529, A63 (2011)

8. Silva Aguirre, V., Basu, S., Brandão, I. M., et al., ApJ 769, 141 (2013) 